\title{
Hirschberg J, Szende T, Koltai PJ, Illényi A: The pediatric airway. Cry, stridor, and cough
}

\author{
Plural Publishing, San Diego, Toronto, Brisbane, 2009, ISBN-13: 978-1-59756-086-3
}

\author{
Istvan Sziklai
}

Published online: 19 November 2009

(C) Springer-Verlag 2009

This monograph collects relevant information on the pediatric airway as to examination, pathology and treatment of airway abnormalities. Besides the current diagnostic techniques used to establish the localization, degree and form of morphological and/or functional disorders, this monograph also provides us with an excellent and detailed description of sound produced by the pathological airway.

Acoustic analysis of cry, cough and stridor is correlated with different types of airway diseases.

A special value of the book is that the diagnostic modality is a noninvasive method. The authors have executed a pioneering work on infant cry analysis to assess hearing impairment and to follow-up and decide the success of the rehabilitation provided.

The observations obtained on cry, stridor and cough in 800 children during the last 45 years by Hirschberg et al. have been gradually refined using advanced technologies for acoustic examinations from sound spectral analysis through digital signal processing until recent nasometric subtraction sound analysis.

The determinant features of the acoustic phenomena of airway sound production are fundamental frequency, formant structure, pitch, loudness, melody and noise components. The first six chapters prepare the reader for understanding acoustic analysis of the sound produced by the infant pathological airway. Chapter 6 describes the acoustic characteristics of different types of crying, coughing and stridor. Nineteen different pathological crytypes, 22 pharyngeal and laryngeal stridor-types and 12

\section{Sziklai $(\square)$}

Department of Otorhinolaryngology and Head and Neck Surgery,

University of Debrecen, Debrecen, Hungary

e-mail: isziklai@dote.hu cough-types are distinguished and demonstrated by sonogram records. The pathology-related diagnostic measures for objective establishment of the pediatric airway abnormalities are endoscopic techniques, mainly laryngobronchoscopy, imaging techniques, electrophysiological examinations, e.g. electroencephalography, electronystagmography, laryngeal electromyography. Suggested algorithms for pediatric airway evaluation also include genetic workup and counseling besides acoustic and endoscopic procedures to confirm the underlying abnormality.

The airway pathologies are systematically overviewed from the oral cavity and nose down to the alveoli in Chap. 7. Each airway abnormality is demonstrated by sonograms as to the acoustics of the disorders and endoscopic pictures. Treatment options for different diseases are discussed in the light of the authors' own experiences. Especially useful descriptions are provided for surgical treatment of laryngeal and tracheal stenoses. The procedures are illustrated by drawings. It is emphasized that acoustic analyses of irregular sounds can be utilized for diagnosis, establishing etiology of the airway disorder, selection of optimal treatment option and follow-up. Verification of the diagnosis should, however, always be performed by endoscopic techniques, CT, MRI or neurologic methods.

Keynote messages are stressed as "Key point" in each chapter separated from the plain text. A CD accompanies the monograph and contains images and sound tracks of different types of cry, cough and stridor records.

Black and white photographs illustrate the book and acceptable for sonograms. The quality of the endoscopic pictures had been better in color.

The references section contains more than 700 citations.

Among the many monographs on pediatric airway, this is unique in acoustic analysis of vocalization due to different pediatric airway diseases and malformations. 